\title{
ESR1 gene amplification in an Iranian population with early onset invasive ductal breast carcinoma
}

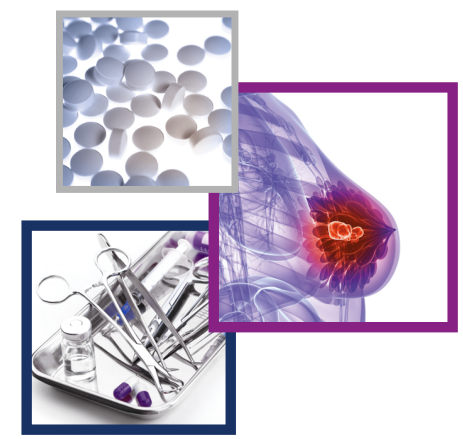

\author{
Asaad Azarnezhad ${ }^{1}$, Mina Tabrizi ${ }^{1}$, Morteza Atri² \& Parvin Mehdipour*,1 \\ ${ }^{1}$ Department of Medical Genetics, School of Medicine, Tehran University of Medical Sciences, Tehran, Iran \\ ${ }^{2}$ Department of Surgery, Cancer Institute, Faculty of Medicine, Tehran University of Medical Sciences, Tehran, Iran \\ *Author for correspondence: Tel.: +98 2188 30100; mehdipor@tums.ac.ir
}

\section{Practice points}

- Breast cancer (BC) is a genetically heterogeneous disease in which clinical presentations are varied.

- The product of ESR1 gene is essential for sexual development and reproductive function and its deficiency is involved in a variety of diseases including breast cancer, endometrial cancer and osteoporosis.

- ER- $\alpha$ expression is considerably higher in premalignant breast disease and malignant BC.

- ER- $\alpha$ high expression status is a good prognostic indicator, and immunohistochemical detection of estrogen receptor expression is routinely used in making decisions on hormonal therapy for BC.

- Gene amplification plays a major role in gene overexpression, therefore, ESR1 amplification may also play as a crucial prognostic and reliable predictor of clinical response to treatment of BC.

- qPCR is a rapid, cost-effective, highly sensitive and applicable to small tissue samples for quantification of gene dosage.

- Our findings showed that the modified SYBR Green qPCR can be applied for relative quantification of ESR1 gene copy number with high sensitivity and specificity compared with immunohistochemistry. However, our data need to be confirmed by fluorescence in situ hybridization.

- Our data disclosed a significant relationship between ESR1 amplification and ER- $\alpha$ expression and positive response to tamoxifen.

- ESR1 amplification also showed a correlation with lower tumor grade and lower tumor stage which suggests a likely prognostic value.

Aim: The study aimed to evaluate a modified SYBR Green qPCR method to study the inter-relations of ESR1 amplification to other parameters including ER expression status, clinicopathological features and responsiveness to tamoxifen. Materials \& methods: 35 breast cancer tissues were assessed for ESR1 amplification by a modified qPCR. Results: Amplification of ESR1 was observed in $31.4 \%$ out of 35 samples. ESR1 amplification and overexpression were significantly correlated (Spearman's Rho $=0.658 ; p<0.001$ ). ESR1 amplification was also statistically associated with positive response to tamoxifen $(p=0.0005)$, lower tumor grade $(p=0.027)$ and lower tumor stage $(p=0.005)$. Conclusion: Findings showed a positive correlation between ER amplification and ER- $\alpha$ expression and highlighted the potential clinical value of using SYBR Green qPCR to quantify amplification of ESR1. ESR1 amplification could be used as an indicator of positive response to tamoxifen and might have a clinicopathological significance. However, the qPCR data should be confirmed by fluorescence in situ hybridization.

First draft submitted: 20 October 2017; Accepted for publication: 16 May 2018; Published online: 30 July 2018

Keywords: estrogen receptor $1 \bullet$ gene amplification • overexpression

Breast cancer (BC) is a genetically heterogeneous disease in which clinical presentations are varied [1]. Based on the clinical outcome, it is divided into three major subgroups consisting of ER-positive tumors; HER2-amplified tumors and triple-negative (basal-like) tumors [2,3]. ESR1 is located on the chromosome 6q25 and its product, and ER- $\alpha$ regulates the transcription of about 5\% of the genome genes [4]. ESR1 encodes a ligand-activated transcription factor composed of several important domains for hormone binding, DNA binding and activation of transcription [5]. The product of the ESR1 gene is essential for sexual development and reproductive function, and plays a role in 
other tissues such as bone. Involvement of ESRI has been reported in a variety of diseases including breast cancer, endometrial cancer and osteoporosis [6]. It has been shown that normal breast epithelial cells express ER- $\alpha$ at a low level and an oscillation expression pattern has been observed throughout the menstrual cycle [7]. However, ER- $\alpha$ expression is considerably higher in premalignant breast disease, and more than two-thirds of breast cancers show estrogen receptor expression at the time of diagnosis. Immunohistochemical detection of estrogen receptor expression is routinely used in making decisions on hormonal therapy for breast cancer [8]. ER- $\alpha$ high expression status is a good prognostic indicator which is associated with longer survival in patients receiving endocrine therapy regimes such as tamoxifen and raloxifene [9].

It is thought that gene amplification plays a major role in the regulation of protein expression. Several amplicons have been reported in different types of cancer which have been related to tumor progression, clinical course of cancer or responsiveness to therapy [10]. ESR1 amplification may play as a reliable predictor of clinical response to treatment of $\mathrm{BC}$. It is reported that ESR1 amplification status is an independent and powerful predictor of long-term survival in women with endocrine-responsive early stage breast cancer who received 5 years of tamoxifen [11].

Given the crucial prognostic and predictive information that can be obtained by the analysis of ESR1 amplification, rapid, accurate and reliable methods for its quantification are required. Immunohistochemistry (IHC) assay is presently used as the method of choice for determination of ER status [12]. Nonetheless, it faces some drawbacks such as being semi-quantitative, low-throughput, subjective, lack of uniformity in reagents, storage and criteria used for scoring, considerable interobserver variability [13].

In addition to the importance of ER- $\alpha$ in response to endocrine therapy, the association of ESR 1 amplification status with patients and clinical parameters and its prognostic/predictive values have not been thoroughly studied to date [14]. Based on a report in 2008, the data gathered from different center revealed that ESR1 amplification in $\mathrm{BC}$ is a rare event of unknown clinical significance [15]. The advent of robust, rapid, sensitive and reproducible quantitative PCR (qPCR) encouraged us to design the present study aimed to quantify the amplification frequency of ESRI in an Iranian population with early onset invasive ductal breast carcinoma using a modified SYBR Green qPCR approach, compare the applied qPCR approach with IHC, compare the expression and amplification of ESR and determine the correlation of ESR1 amplification with the patient and clinical factors and response to tamoxifen therapy.

\section{Materials \& methods \\ Patients}

The current study was performed in Tehran, Iran between 2016 and 2017. 35 fresh frozen BC specimens were included in the present study. All the histopathologic diagnoses were performed by pathologists and all the specimens were collected from patients with early onset invasive ductal breast carcinoma. Ten samples taken from a cosmetic breast surgery center were used as the calibrator sample. All cases were histologically classified according to the Bloom-Richardson grading system. Hematoxylin and eosin-stained slides from each case were reviewed to confirm the diagnosis of either invasive ductal carcinoma (IDC) or invasive lobular carcinoma (ILC). Pathology reports were used to extract the ER- $\alpha$ expression information. Clinicopathological characteristics and IHC data were collected from the pathological and medical documents. The present work was performed under the approval of Research Ethics Committee of Tehran University of Medical Science (Tehran, Iran) and according to the Declaration of Helsinki, a signed consent form was taken from each patient. Assessment of response to tamoxifen was performed based on response evaluation criteria in solid tumors.

\section{DNA extraction \& SYBR Green qPCR}

We were blind to ER- $\alpha$ expression status before we obtained the relative copy number of ESR1. Total DNA was extracted from all the fresh frozen BC tissue sections using QIAamp kit (Hilden, Germany) according to the instruction of manufacturer. Quantity/quality of the extracted DNA was measured by NanoDrop 2000 (MA, USA). Assuming that there are not any differences in the quality and quantity of starting DNA and the PCR efficiency of the target gene, we used $\mathrm{E} \mathrm{Cp}$ (gene of interest in calibrator)- $\mathrm{Cp}$ (gene of interest in the case) formula to quantify the relative copy number (RCN) of the target gene [16]. Five tenfold serial dilutions of a DNA sample were prepared and the efficiency of each primer was determined according to $\mathrm{E}=10-1 /$ slope. Target gene was amplified by SYBR Green-based qPCR using primers: 5'- GCCCCCGATACTCTATTCCG - $3^{\prime}$ and 5'CGCTTCGCATTCTTACCTGG -3'. Twenty microliters of PCR reactions containing $10 \mu \mathrm{l}$ SYBR Premix Ex $\mathrm{Taq}^{\mathrm{TM}}$ (Tli RNaseH Plus) (Takara Bio, Japan), $1.5 \mu \mathrm{l}$ DNA (40 ng/ $\left.\mu \mathrm{l}\right), 0.5 \mu \mathrm{l}$ primer (each, $5 \mathrm{pmol}$ ) and $8.5 \mu \mathrm{l}$ 
Table 1. The qPCR data including $C p$ values, $\Delta C t$ and $2^{-\Delta c t}$.

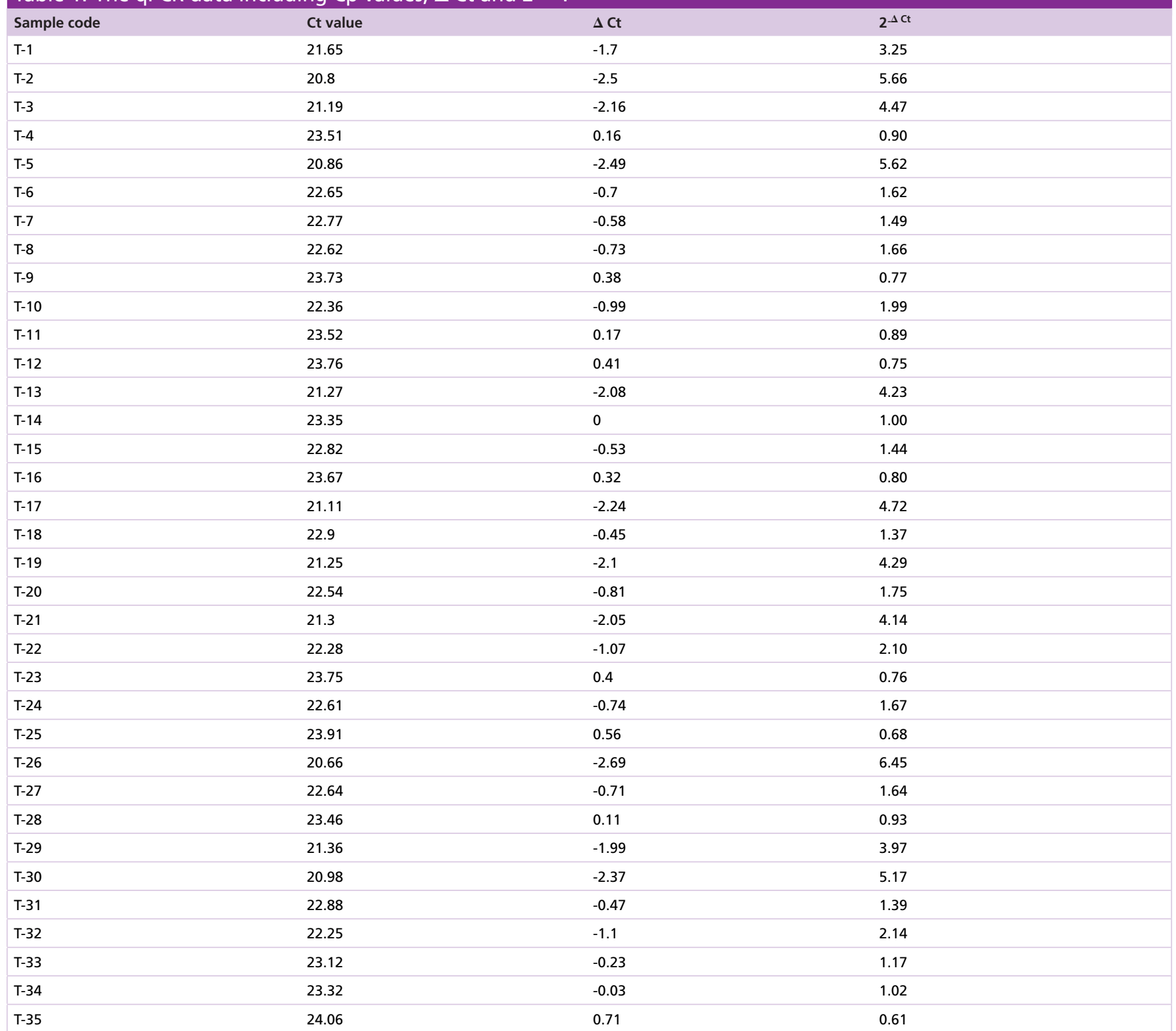

Ct value of pooled normal calibrator was 23.35. As PCR product accumulates, fluorescence signaling increases and rises above background levels at a quantifiable point referred to as the $\mathrm{Ct}$.

double distilled water were run in Rotor-Gene 2000 (Qiagen, Germany) according to the following program: initial heating at $95^{\circ} \mathrm{C}$ for $30 \mathrm{~s}, 40$ cycles of denaturation at $95^{\circ} \mathrm{C}$ for $10 \mathrm{~s}$, annealing and extension at $59^{\circ} \mathrm{C}$ for 30 s. A melting curve was then plotted between 60 and $99^{\circ} \mathrm{C}$ to the analysis of specific amplification of target region. All quantification analyses were performed based on the cycle of a threshold value $(\mathrm{Cp})$. All reactions were run in duplicates and mean Cp was put into RCN calculation. The RCN of ESR1 was determined by qPCR in tumor sample relative to a normal calibrator reference of $\mathrm{CN}=2$ (diploid). The qPCR data ( $\mathrm{Cp}$ values) of the tumor and normal samples are shown in Table 1.

Furthermore, the results of $\mathrm{E} \mathrm{Cp}$ (gene of interest in calibrator)- $\mathrm{Cp}$ (gene of interest in the case) formula were confirmed by using $2^{-\triangle \triangle \mathrm{Ct}}$ quantification method, wherein $H B B$ and $R P L P O$ genes were amplified as internal control and relative-fold differences of target were calculated. A forward primer: GCTTCTGACACAACTGTGTTCACTAGC and a reverse primer: CACCAACTTCATCCACGTTCACC for a $120 \mathrm{bp}$ fragment of $H B B$ and 


\begin{tabular}{|c|c|c|c|}
\hline Parameter & Frequency (\%) & Parameter & Frequency (\%) \\
\hline Age & & ER status (IHC) & \\
\hline$<30$ & $9(25.7)$ & Positive & $16(45.7)$ \\
\hline$>30$ & $26(74.3)$ & Negative & $19(54.3)$ \\
\hline Tumor size, cm & & PR status & \\
\hline$>2$ & $11(31.4)$ & Positive & $13(37.1)$ \\
\hline$<2$ & $24(68.6)$ & Negative & $22(62.9)$ \\
\hline Tumor stage & & ALN metastasis & \\
\hline I & $1(2.9)$ & Yes & $21(60)$ \\
\hline II & $17(48.6)$ & No & $14(40)$ \\
\hline III & $17(48.6)$ & & \\
\hline Tumor grade & & Family history & \\
\hline I & $9(25.71)$ & Positive & $14(40)$ \\
\hline II & $11(31.42)$ & Negative & $21(60)$ \\
\hline III & $15(42.85)$ & & \\
\hline
\end{tabular}

a forward primer: TGTGGCCTTCTACCTTACTG and reverse primer: TGGGTCTCTTGTCATTTCTC for $R P L P O$ was used to amplify the target regions.

Immunohistochemical analysis

Immunohistochemical analysis (IHC) was performed according to previously standardized protocols on an automated IHC platform (Dako Techmate 500) and the ASCO/CAP guidelines for IHC [12]. The following primary antibody and corresponding dilution of ER (clone 1D5; 1:100) were used (DakoCytomation, Glostrup, Denmark). Slides were assessed by quantitative image analysis using the Aperio Image Analysis toolbox (Leica Biosystems, Nussloch, Germany). Staining intensity and percentage of positive nuclei were recorded after manually segmenting tumor from the adjacent stroma. Tumors with ER scores greater than 3 or positive nuclei greater than $1 \%$ were considered estrogen receptor positive.

\section{Statistical analysis}

The cut-off point of amplification was established by plotting qPCR results compared with IHC data in receiver operating characteristic (ROC) curve. The correlation between applied qPCR and IHC was determined by calculating the Spearman's Rho. The chi-square test was used to analyze associations of gene amplification with clinicopathological characteristics and responsiveness to tamoxifen. All p-values were two-sided and points less than 0.05 were considered statistically significant. SPSS for Windows (version 16.0) was used for statistical analyses.

\section{Results}

\section{Patients \& clinicopathological data}

The oldest and youngest participants were 49 and 16 years old, respectively, and the mean age \pm SD was $37.54 \pm 8.83$ years. All the collected clinicopathological parameters are demonstrated in Table 2. Regardless the ER- $\alpha$ expression status, 35 IDC tumor samples were investigated for gene amplification of ESR1 by using qPCR. Flowchart for patients' selection in this study has been illustrated in Figure 1.

\section{Correlation of ESR1 status with clinicopathological features}

Association of ESR1 status with tumor and patient's characters is shown in Table 3. ESR1 amplification is statistically associated with ER- $\alpha$ expression $(\mathrm{p}<0.001)$, positive response to tamoxifen $(\mathrm{p}=0.0005)$, lower tumor grade $(\mathrm{p}=0.027)$ and lower tumor stage $(\mathrm{p}=0.005)$. However, there are no significant relations between ESR1 status and age of diagnosis, tumor grade, tumor size, tumor stage, PR status and metastatic status. Spearman's Rho values $>0.50$ are indicative of a positive relationship between compared groups.

\section{Correlation of qPCR with IHC}

qPCR results were compared with IHC data obtained from medical documents of patients. The detection performance or accuracy of applied SYBR Green qPCR test compared with IHC was evaluated using ROC curve analysis. The area under the ROC curve (AUC) is a measure of how well the applied qPCR test can precisely discriminate 


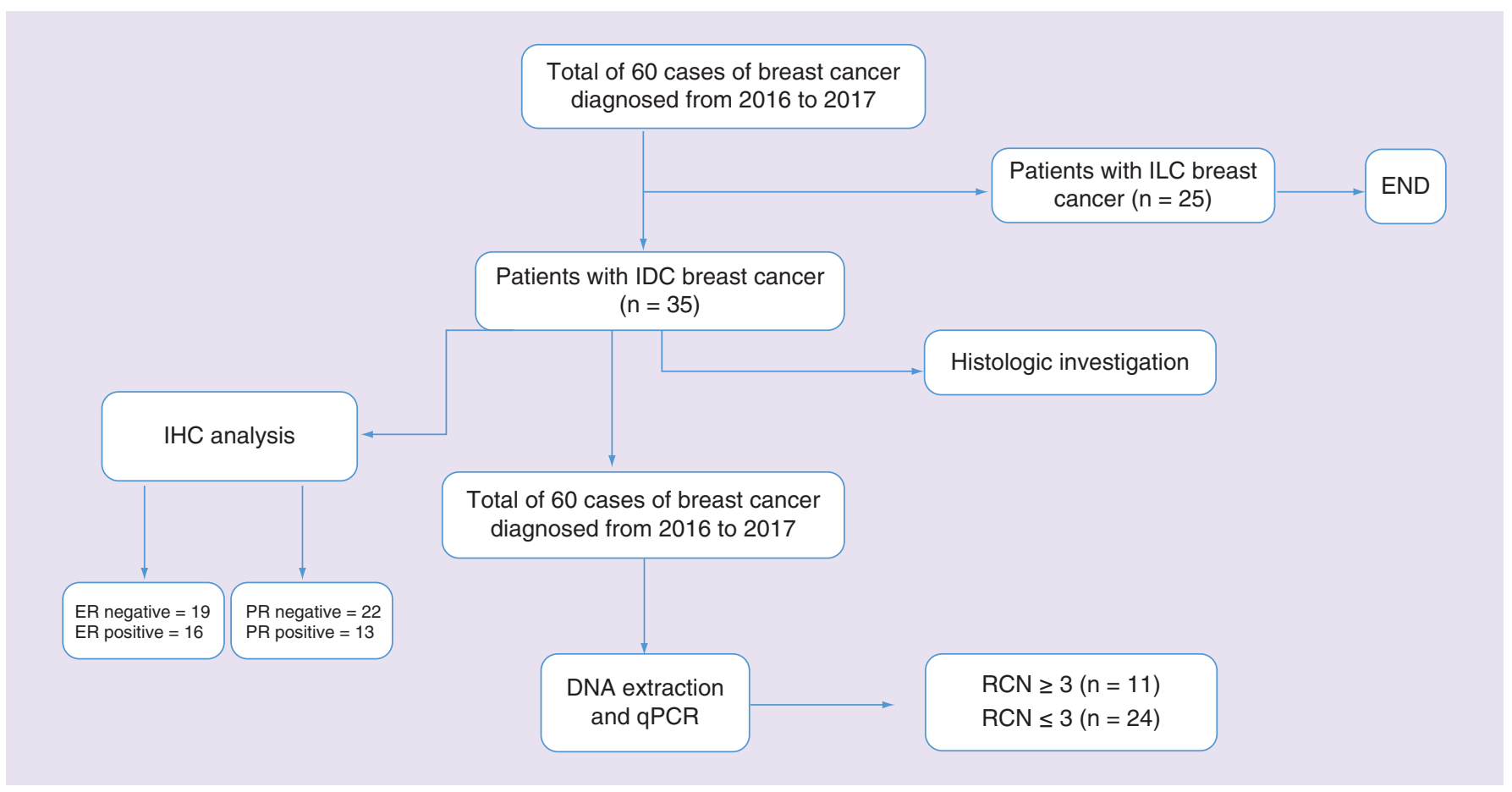

Figure 1. Flow chart for patients' selection in this study.

ER: Estrogen receptor; IDC: Invasive ductal carcinoma; IHC: Immunohistochemistry; ILC: Invasive lobular carcinoma; PR: Progesterone receptor; RCN: Relative copy number.

amplified from unamplified conditions. AUC for detection ESR1 amplification was $0.822(\mathrm{p}=0.001)$ which is indicative of excellent sensitivity and specificity. Based on the ROC curve analysis, RCN $\geq 3$ was considered as the amplification point of target genes (Figure 2). Spearman's Rho and concordance between qPCR and IHC for detection of ESR1 status was 0.656 and $82.85 \%$ (29/35), respectively. As protein expression is measured in IHC and gene amplification in qPCR, a correlation between qPCR and IHC is also an indication of correlation between gene copy number and protein expression.

\section{Sensitivity, specificity, positive \& negative predictive values}

The current study was a diagnostic type. Therefore, the sensitivity, specificity, negative predictive value and positive predictive value values were calculated for an applied qPCR method, using IHC as the gold standard. These values were measured to evaluate the performance of applied qPCR to detect the amplification of ESR1. Sensitivity, specificity, negative predictive value and positive predictive value were $75,89.47,80.95$ and $85.71 \%$ respectively.

\section{Discussion}

The aims of this study were to analyze the frequency of ESR1 amplification by a modified SYBR Green qPCR, to compare these amplifications with IHC data, to associate these amplifications with clinicopathological features and to correlate the amplification status of ESRI with responsiveness to tamoxifen. Our data disclosed that ESRI is amplified in $31.4 \%$ of the included population affected by early onset invasive ductal breast carcinoma. However, the current study has limitations. The sample size of this study is relatively small. Larger studies are required to determine the percentage of ESR1 gene amplification in $\mathrm{BC}$ definitively. According to our results, the frequency of ESR1 gene amplification differed than that for reports of other geographical patients with BC. This suggests that the contribution of ESRI gene amplification to the pathogenesis and progression of $\mathrm{BC}$ is probably different between ethnicities. Technical issues and lack of standards may also contribute to the discrepant findings. Comparison of qPCR results with IHC data showed that applied qPCR could determine the ESR1 status more rapidly in a time/cost-effective manner with high sensitivity and specificity. Our findings also showed a significant correlation between ESR1 amplification and ER expression, tamoxifen-responsiveness, lower tumor grade and stage. 
Table 3. Patient and tumor characteristics according to ESR1 status.

\begin{tabular}{|c|c|c|c|}
\hline Patient and tumor characteristics & ESR1 amplified number $(\%)^{\dagger}$ & ESR1 unamplified number (\%) & p-value \\
\hline All & $11(31.4)$ & $24(68.8)$ & - \\
\hline \multicolumn{3}{|l|}{ Age at diagnosis } & 0.752 \\
\hline$>30$ & $4(28.6)$ & $5(23.8)$ & \\
\hline \multicolumn{3}{|l|}{ Tumor size, $\mathrm{cm}$} & 0.298 \\
\hline$>2$ & $11(78.6)$ & $13(61.9)$ & \\
\hline$<2$ & $3(21.4)$ & $8(38.1)$ & \\
\hline Grade 3 & $4(28.6)$ & $14(66.7)$ & \\
\hline \multicolumn{3}{|l|}{ Tumor stage } & 0.005 \\
\hline Stage $1+2$ & $12(85.7)$ & $8(38.1)$ & \\
\hline Stage 3 & $2(14.3)$ & $13(61.9)$ & \\
\hline \multicolumn{3}{|l|}{ ER status } & $<0.001$ \\
\hline Negative & $2(14.3)$ & $17(81)$ & \\
\hline Positive & $12(85.7)$ & $4(19)$ & \\
\hline Negative & $6(42.9)$ & $8(38.1)$ & \\
\hline Positive & $8(57.1)$ & $13(61.9)$ & \\
\hline \multicolumn{3}{|l|}{ Family history } & 0.067 \\
\hline Negative & $11(78.6)$ & $10(47.6)$ & \\
\hline Positive & $3(21.4)$ & $11(52.4)$ & \\
\hline \multicolumn{3}{|l|}{ Tam responsiveness ${ }^{\ddagger}$} & 0.0005 \\
\hline Positive & $11(78.6)$ & $4(19)$ & \\
\hline Negative & $3(21.4)$ & $17(81)$ & \\
\hline
\end{tabular}

Given the ESR1 gene amplification, a large volume of studies on this topic have been published, and a wide range of inconsistent results have been reported. Although there are many reports in which ESR1 is amplified in 20.6, $4.2,2,11.3$ and $0 \%$, the reported prevalence of this alteration in breast cancer ranges from 0 to $75 \%$, proposing that ESR1 copy number analysis is prevented by technical and interpreter issues [4,8,9,14,17-19]. About $70 \%$ of breast cancers are ER positive. Gene amplification is the main mechanism for cancer cells to increase the expression of gene products that provide them with a growth or survival advantage. Therefore, amplification may be a potential therapeutic target as same as the example of $E R B B 2$ and the success of trastuzumab (Herceptin $\cap$ ) for the treatment of Her2-amplified or Her2-overexpressing breast cancers [20]. We found a significant correlation between ESR1 amplification and ER overexpression. The vast majority of the amplified tumors were also ER-overexpressed which is consistent with previous reports [9,18]. Accordingly, it was found that ESRI amplification was significantly related to positive response to tamoxifen, therefore the response to tamoxifen could also rely on ESR1 amplification. Given the considerable association between ESRI amplification and positive estrogen receptor, IHC not only validates our experimental approach but also demonstrates the strong functional importance of ESR1 gene amplification. Considering the results of the current study and those of the previous report, the mechanism of ER overexpression may be dualistic, consisting of gene amplification as well as activation at the transcriptional level [19,21]. As a result, some cases of inconsistencies could be due to overexpression at the transcription level, not the DNA level, and this could be considered as the limitation of the study when the qPCR and IHC data are compared. Therefore, technical errors might be the reason of low concordance between qPCR and IHC and decrease in sensitivity, specificity, positive and negative predictive values of applied qPCR approach. According to a report, it is more informative to assess the HER-2/neu gene amplification than IHC overexpression. Failure to detect fluorescence in situ hybridization (FISH)-amplified (IHC-negative) cases would have an adverse effect on the survival of these patients. On the other hand, IHC overexpression tumors without gene amplification appear to belong to a better prognostic group, and failure to detect them would probably not have a negative impact on the survival of these 


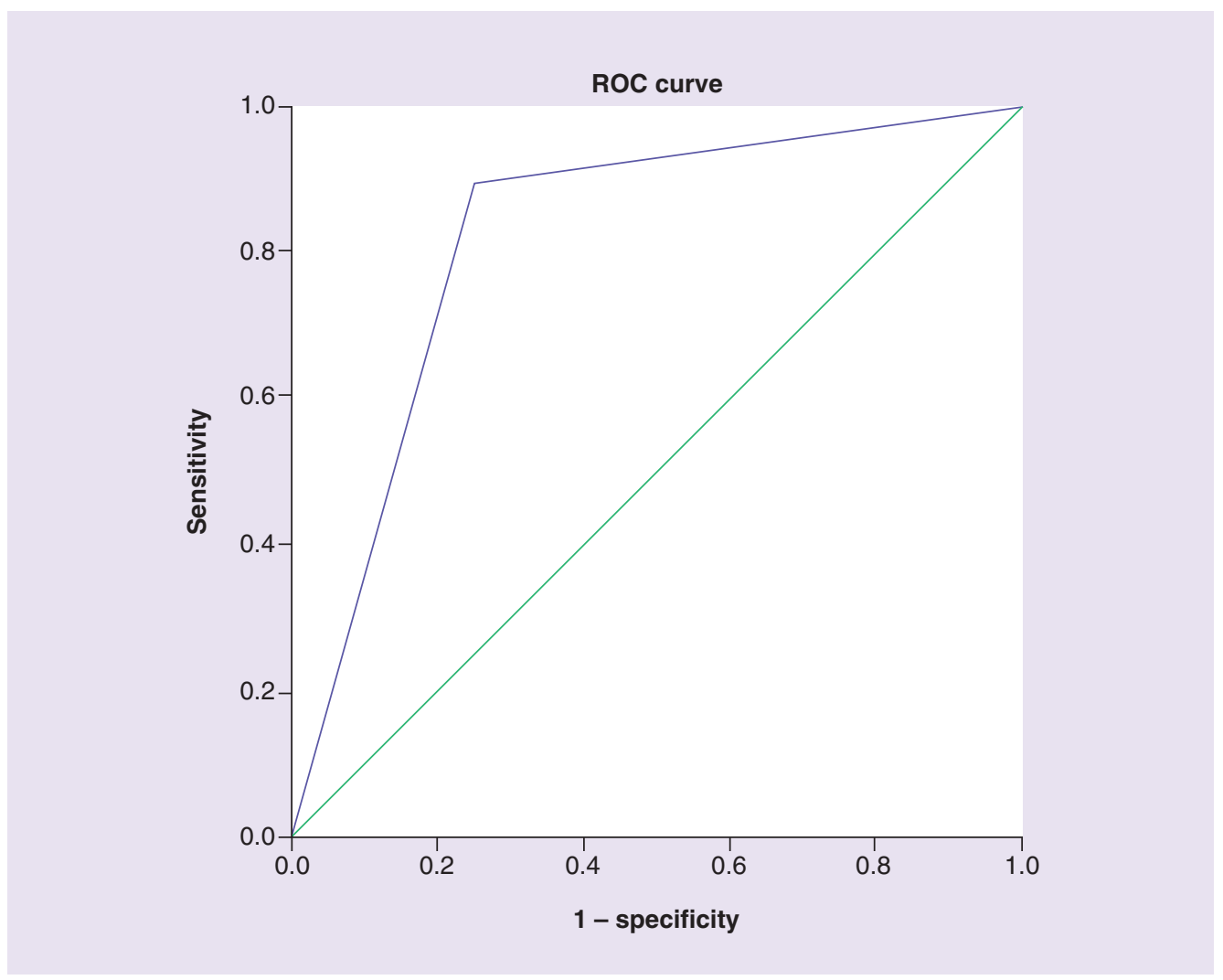

Figure 2. Receiver-operating characteristic curve. This graphical plot illustrates the diagnostic ability including sensitivity and specificity of applied SYBR Green qPCR approach. Relative copy number $\geq 3$ was set for ROC analysis, wherein, $A \cup C=0.822, p=0.001$ and $95 \% \mathrm{Cl}: 0.672-0.973$. These data are indicative of an excellent diagnostic ability of the applied qPCR in comparison to immunohistochemistry. The blue line represents the plot of sensitivity versus 1 -specifity and the green line is a tangent line whose slope at a cut-point gives the likelihood ratio for that value of the test.

ROC: Receiver-operating characteristic.

women [22]. The same scenario may also be the reason of nonresponsiveness of ER- $\alpha$-positive tumors without ESR1 amplification to tamoxifen or other antiestrogen therapy. However, this is just a hypothesis and must be evaluated, ideally in an experimental setting. Unfortunately, recurrences still do occur in $30-40 \%$ of ER positive breast cancer patients, and ESR1 or other biomarkers amplification may be due to the etiology of nonresponsiveness to tamoxifen. Sanaz Tabarestani and colleagues showed that CCND1 amplification may serve as a useful biomarker for hormone responsiveness [23].

Microarray studies on tumors and breast cancer cell lines have shown that 40-61\% of overexpressed genes have also been genetically amplified and the findings have provided evidences that widespread DNA copy number alteration can lead directly to global deregulation of gene expression [24]. The main purpose of the current study was to compare ESR1 amplification with its expression status and to show how efficiently qPCR can determine ESRI amplification as a complementary tool to other techniques such as FISH. A broad range of techniques such as array comparative genomic hybridization, chromogenic in situ hybridization (CISH), FISH and qPCR has been applied for quantitation of ESR copy number $[4,9,15,25]$. Given the critical role of ER expression for breast cancer therapy and the importance of gene amplification in overexpression, need to a rapid, cost-benefit and accurate method for ESR1 profiling is highlighted. Although each technique has its advantages and disadvantages, a modified qPCR approach was developed to determine the ESR1 copy number. The SYBR Green and TaqMan assays are usual chemistries for real-time PCR. Owing to its simple design, easy setup, and low cost, SYBR Green is predominantly used for the detection and quantification of nucleic acids. Absolute and relative quantification are two different PCR-based methods of presenting quantitative gene copy number. Absolute quantification is based on the calculation of the copy number of a target gene by relating the PCR signal to a standard curve. However, 
relative gene copy number quantification presents the data of target gene relative to some calibrator or internal control gene. According to our knowledge, absolute quantification or relative quantification based on hybridization probes has previously been applied to gene copy number quantification. Nonspecific binding of the SYBR Green dye to the nontarget double-strand DNA molecule can lead to false-positive results. However, any limitations in the specificity of dsDNA dyes can be overcome by using postamplification melting curves or choosing high fluorescence acquisition temperatures during amplification and good primer design and optimization of real-time PCR. The most famous qPCR strategies are based on hybridization probes, but they are still expensive and hard to be optimized.

In contrast with previously reported qPCR methods which have been based on hybridization probes, a modified SYBR Green-based qPCR approach was used to relatively quantify the copy number of ESR1. The common method of relative quantification is based on $2^{-\Delta \Delta \mathrm{Ct}}$ formulae. Assuming that there are not any differences in the quality and quantity of starting DNA and the PCR efficiency of the target gene, a modified SYBR Green qPCR based on $2 \mathrm{Cp}$ (gene of interest in calibrator)-Cp(gene of interest in the case) formula was used to the RCN of ESR1. In this approach, internal reference gene was not assessed so that the number of required reactions is decreased considerably and consequently time and cost. Due to sensitivity to contamination with normal stromal cells, DNA extraction was performed from biopsied cells of microdissected tissue sections containing more than $85 \%$ tumor cells. Besides, the accuracy of the applied formula was confirmed using the common method of relative quantification, $2^{-\triangle \triangle \mathrm{Ct}}$, in which $H B B$ and $R P L P O$ genes were amplified as the internal single copy reference genes. The relationship between $2^{-\Delta \mathrm{ct}}$ and $2^{-\Delta \Delta \mathrm{ct}}$ methods in determining the ESR1 copy number of is illustrated in Figure 3. The linear relationship between the results of the two methods indicated the accuracy of the modified $2^{-\Delta c t}$ approach used in this study. Although FISH is the gold standard way to profile gene copy number and is more objective and quantitative than immunohistochemical scoring, it is more expensive, fluorescence signals fade within a few weeks and the FISH assay can be interpreted only by well-trained personnel.

Our data revealed $82.85 \%$ concordance and significant correlation between $\mathrm{QPCR}$ and IHC; therefore the data presented suggest that the ESR1 amplification status might be determined by qPCR and could be used complementarily in addition to IHC to potentially compensate limitations of IHC, and in making decisions on hormonal therapy for breast cancer. Concordance between $\mathrm{PPCR}$ and IHC has been reported in different kinds of literature in which the value of correlation has been varied in a range of 80-95\% [26]. Consistent with our findings, IHC for ER and PR revealed a high concordance with RT-qPCR (Spearman's Rho $=0.82 ; \mathrm{p}<0.0001$ ) in a recent study. However, RT-qPCR displayed a broader dynamic range and higher sensitivity than IHC [19].

Association of ESR1 amplification status with patients and tumors' characteristics was also assessed as a further aim. As we performed the current work on a specific type of breast cancer, early onset invasive ductal carcinoma, this essential inclusion criterion for the study population led to the limitation of sample size. However, this point could be considered as the limitation of the study. Correlation of ESR1 amplification with clinicopathological parameters has been studied. Our findings showed that ESR1 amplification is considerably related to lower tumor grade and stage. These findings would be consistent with a very early, if not initializing, a role for ER amplification for a subset of breast cancers. Thus, it might be concluded that ESR1 amplification status shows a useful prognostic marker and suggests that $E S R 1$ amplification may be a common mechanism in individuals with BC. It is reported that epigenetic signature within the ESR1 gene CpG island is changed in a series of nonmalignant and preneoplastic breast tissues [27]. Although these findings have been confirmed in a small number of reports, there are a variety of studies with contradictory results in which ESR1 status has been linked with higher tumor grade, stage, size, nodal metastasis and poorer outcome $[4,8,9,14,28]$. The prognostic or predictive power of the ESR1 amplification status determined by qPCR was compared with the prognostic or predictive power of the ER- $\alpha$ status determined by IHC. Data showed that ER- $\alpha$ status was significantly associated with tumor grade $(p=0.005)$, tumor stage $(\mathrm{p}=0.004)$ and positive response to tamoxifen $(\mathrm{p}=0.000000024)$.

We are aware of several limitations of this study, including small sample size, qPCR sensitivity to DNA quality and quantity, falling qPCR results between integers and therefore more difficult interpretation, sensitive to finding a true cut-off point of amplification, qPCR sensitivity to mosaicism, and contamination with a large number of normal stromal cells. To avoid these limitations as much as possible, different filtering criteria and steps are proposed to be considered in the process of work design including microdissection of tumor tissue to cut the area containing $>85 \%$ cancer cells, primer design and in silico quality control, empirical validation of primers, preparation of gDNA dilution series for qPCR assay evaluation and melt curve analysis. Also, the study of a larger population might result in more validated data. The accuracy of the applied formula is also suggested to 


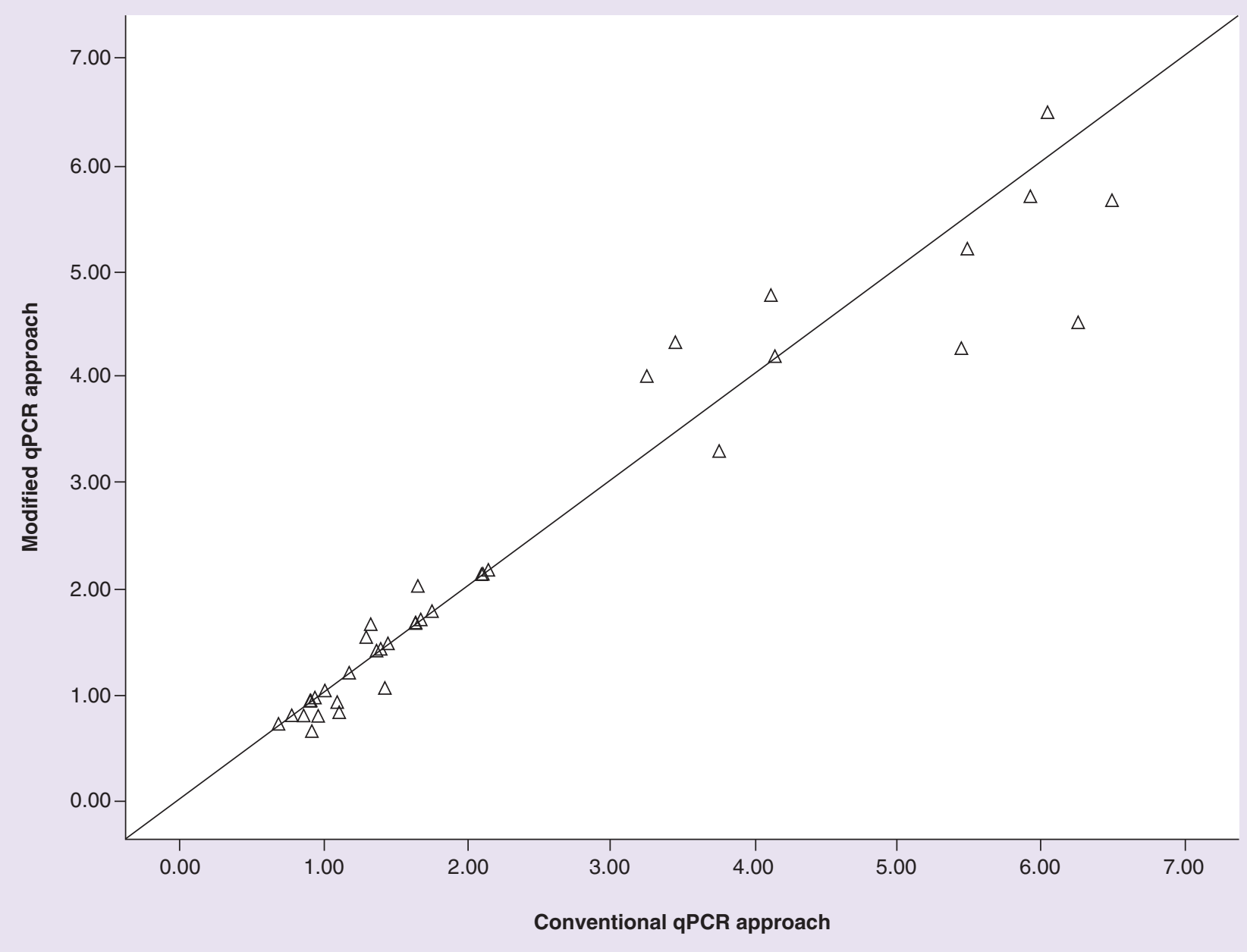

Figure 3. The relationship between $2^{-\Delta c t}$ (modified qPCR approach) and $2^{-\Delta \Delta c t}$ (conventional qPCR approach) methods in determining the copy number of the ESR1 gene. In this chart, the linear relationship between the results of the two methods indicates the high accuracy of the modified $2^{-\Delta c t}$ approach used in the current study. The numbers at the vertical axes represent the relative copy number of ESR1 determined by the modified qPCR approach and the numbers at the horizontal axes represent the relative copy number of ESR1 determined by the conventional qPCR approach. R Sq Linear is 0.92 .

be confirmed using FISH and this could be mentioned as another limitation of the study. Currently, emphasis must be put on standardization of procedures, internal and external quality control assessment, and competency evaluation of already existing methods to ensure accurate, reliable and clinically meaningful test results. However, development of new cost/time benefit, robust and accurate diagnostic assays is also required to be encouraged.

\section{Conclusion}

The present study highlights the potential clinical value of using SYBR Green qPCR to quantify amplification of ESR1. Although qPCR provides quick and robust results, a standardized and validated definition of a cut-off point of amplification needs to be investigated before its potential use in daily practice. The accuracy of the applied qPCR is also suggested to be confirmed using FISH. Our data disclosed a significant relationship between ESR1 amplification and ER- $\alpha$ expression, positive response to tamoxifen, lower tumor grade and lower tumor stage. However, there were inconsistencies between ESRI amplification and IHC data and determination of the ESRI amplification status may not be confidently suggested to be an alternative to ER- $\alpha$ status determined by IHC. Furthermore, more studies with larger sample size are suggested to reproduce and confirm our findings. The aim of strategically BC management would be achievable through unmasking the functional event at tumor level. Nevertheless, by considering the complementary dual assay such as expression status of ESR1 and Her2-neu, could 
be translatable to the target- and endocrine-based therapy. Combinational therapy also relies on expression mode of ESR1 and Her2-neu including $\mathrm{Er}+/ \mathrm{Her} 2-, \mathrm{Er}+/ \mathrm{Her} 2+$ for updating the approach of clinical management for $\mathrm{BC}$ patients including the metastatic BC. Besides, exploring the role of the available biomarker(s) for the prevention, assessment, and management guidelines of breast cancer can be considered as a future perspective.

\section{Acknowledgements}

The authors would like to show their gratitude to the patients and the participants as normal controls in this study. This article was extracted from a part of the PhD dissertation on medical genetics by A Azarnezhad, supervised by P Mehdipour and M Tabrizi.

Financial \& competing interests disclosure

This research was supported by Tehran University of Medical Sciences (Tehran, Iran) under the registered Research Grant (number 31224). The authors have no other relevant affiliations or financial involvement with any organization or entity with a financial interest in or financial conflict with the subject matter or materials discussed in the manuscript apart from those disclosed.

No writing assistance was utilized in the production of this manuscript.

Ethical conduct of research

The authors state that they have obtained appropriate institutional review board approval or have followed the principles outlined in the Declaration of Helsinki for all human or animal experimental investigations. In addition, for investigations involving human subjects, informed consent has been obtained from the participants involved.

\section{Open access}

This work is licensed under the Attribution-NonCommercial-NoDerivatives 4.0 Unported License. To view a copy of this license, visit http://creativecommons.org/licenses/by-nc-nd/4.0/

\section{References}

1. Lakhani SR. WHO Classification of Tumours of the Breast. International Agency for Research on Cancer (2012). https://books.google.com/books/about/WHO_Classification_of_Tumours_of_the_Bre.html?id=J8qipwAACAAJ

2. Hashemian AH, Hajizadeh E, Kazemnejad A, Atri M, Mehdipour P. Penetrance of BRCA1/BRCA2 specific gene mutations in Iranian women with breast cancer. Saudi Med. J. 30(1), 41-44 (2009).

3. Kwei KA, Kung Y, Salari K, Holcomb IN, Pollack JR. Genomic instability in breast cancer: pathogenesis and clinical implications. Mol. Oncol. 4(3), 255-266 (2010).

4. Moelans CB, Monsuur HN, De Pinth JH, Radersma RD, De Weger RA, van Diest PJ. ESR1 amplification is rare in breast cancer and is associated with high grade and high proliferation: a multiplex ligation-dependent probe amplification study. Anal. Cell. Pathol. (Amst.) 33(1), 13-18 (2010).

5. Ooi A, Inokuchi M, Harada S et al. Gene amplification of ESR1 in breast cancers: fact or fiction? A fluorescence in situ hybridization and multiplex ligation-dependent probe amplification study. J. Pathol. 227(1), 8-16 (2012).

6. Lin C-H, Liu JM, Lu Y-S et al. Clinical significance of ESR1 gene copy number changes in breast cancer as measured by fluorescence in situ hybridisation. J. Clin. Pathol. 66(2), 140-145 (2013).

7. Markopoulos C, Berger U, Wilson P, Gazet J-C, Coombes RC. Estrogen receptor content of normal breast cells and breast carcinomas throughout the menstrual cycle. Br. Med. J. (Clin. Res. Ed.) 296(6633), 1349-1351 (1988).

8. Holst F, Stahl PR, Ruiz C et al. Estrogen receptor $\alpha$ (ESR1) gene amplification is frequent in breast cancer. Nat. Genet. 39(5), 655-660 (2007).

9. Tomita S, Zhang Z, Nakano M et al. Estrogen receptor $\alpha$ gene ESR1 amplification may predict endocrine therapy responsiveness in breast cancer patients. Cancer Sci. 100(6), 1012-1017 (2009).

10. Pinkel D, Albertson DG. Array comparative genomic hybridization and its applications in cancer. Nat. Genet. 37(6s), S11-S17 (2005).

11. Singer CF, Holst F, Steurer $S$ et al. Estrogen receptor alpha (ESR1) gene amplification status and clinical outcome in tamoxifen-treated postmenopausal patients with endocrine-responsive early breast cancer: an analysis of the prospective ABCSG-6 trial. J. Clin. Oncol. 30(Suppl. 15), 10501-10501 (2012).

12. Hammond MEH, Hayes DF, Dowsett M et al. American Society of Clinical Oncology/College of American Pathologists guideline recommendations for immunohistochemical testing of estrogen and progesterone receptors in breast cancer (unabridged version). Arch. Pathol. Lab. Med. 134(7), e48-e72 (2010).

13. Furrer D, Sanschagrin F, Jacob S, Diorio C. Advantages and disadvantages of technologies for HER2 testing in breast cancer specimens. Am. J. Clin. Pathol. 144(5), 686-703 (2015). 
14. Pentheroudakis G, Kotoula V, Eleftheraki AG et al. Prognostic significance of ESR1 gene amplification, mRNA/protein expression and functional profiles in high-risk early breast cancer: a translational study of the Hellenic Cooperative Oncology Group (HeCOG). PLoS ONE 8(7), e70634 (2013).

15. Brown LA, Hoog J, Chin S-F et al. ESR1 gene amplification in breast cancer: a common phenomenon? Nat. Genet. 40(7), 806-807 (2008).

16. Frank SG, Bernard PS. Profiling breast cancer using real-time quantitative PCR. In: Rapid Cycle Real-Time PCR - Methods and Applications, Springer, Berlin, Heidelberg, Germany. 95-106 (2004).

17. Chen J-R, Hsieh T-Y, Chen H-Y et al. Absence of estrogen receptor $\alpha$ (ESR1) gene amplification in a series of breast cancers in Taiwan. Virchows Arch. 464(6), 689-699 (2014).

18. Holst F. Estrogen receptor alpha gene amplification in breast cancer: 25 years of debate. World J. Clin. Oncol. 7(2), 160-173 (2016).

19. Sinn H-P, Schneeweiss A, Keller M et al. Comparison of immunohistochemistry with PCR for assessment of ER, PR, and Ki-67 and prediction of pathological complete response in breast cancer. BMC Cancer 17(1), 124 (2017).

20. Pegram MD, Lipton A, Hayes DF et al. Phase II study of receptor-enhanced chemosensitivity using recombinant humanized anti-p185HER2/neu monoclonal antibody plus cisplatin in patients with HER2/neu-overexpressing metastatic breast cancer refractory to chemotherapy treatment. J. Clin. Oncol. 16(8), 2659-2671 (1998).

21. Rahman MT, Nakayama K, Rahman M et al. ESR1 gene amplification in endometrial carcinomas: a clinicopathological analysis. Anticancer Res. 33(9), 3775-3781 (2013).

22. Sauer T, Wiedswang G, Boudjema G, Christensen H, Kåresen R. Assessment of HER-2/neu overexpression and/or gene amplification in breast carcinomas: should in situ hybridization be the method of choice? APMIS 111(3), 444-450 (2003).

23. Tabarestani S, Ghaderian SMH, Rezvani $\mathrm{H}$ et al. Prognostic and predictive value of copy number alterations in invasive breast cancer as determined by multiplex ligation-dependent probe amplification. Cell. Oncol. (Dordr.) 37(2), 107-118 (2014).

24. Pollack JR, Sørlie T, Perou CM et al. Microarray analysis reveals a major direct role of DNA copy number alteration in the transcriptional program of human breast tumors. Proc. Natl Acad. Sci. USA 99(20), 12963-12968 (2002).

25. Albertson DG. Conflicting evidence on the frequency of ESR1 amplification in breast cancer. Nat. Genet. 40(7), 821-822 (2008).

26. Rodriguez C, Suciu V, Poterie A et al. Concordance between HER-2 status determined by qPCR in fine needle aspiration cytology (FNAC) samples compared with IHC and FISH in core needle biopsy (CNB) or surgical specimens in breast cancer patients. Mol. Oncol. 10(9), 1430-1436 (2016).

27. Daraei A, Izadi P, Khorasani G et al. Epigenetic changes of the ESR1 gene in breast tissue of healthy women: a missing link with breast cancer risk factors. Genet. Test. Mol. Biomarkers 21(8), 464-470 (2017).

28. Nielsen KV, Ejlertsen B, Müller $S$ et al. Amplification of ESR1 may predict resistance to adjuvant tamoxifen in postmenopausal patients with hormone receptor positive breast cancer. Breast Cancer Res. Treat. 127(2), 345-355 (2011). 
